by $x$ and $y$; then if $V \cap L^{2}=0$ we are done by Theorem 1 . If $z \in V \cap L^{2}$ and $z \neq 0$, then $x$ and $z$ still generate $L$. In this case $L=(x)+L^{2}$ so $z$ has the form $z=\sum\left[a_{i} x+h_{i}, b_{i} x+h_{i}^{\prime}\right]$ with $a_{i}, b_{i}$ in $K$ and $h_{i}, h_{i}^{\prime}$ in $L^{2}$. This means that $z$ is in $L^{3}$. But any element of $L^{2}$ is a sum of words in $x$ and $z$ and each word must contain $z$ i.e., $L^{2} \subseteq L^{3}$ contrary to hypothesis.

HARVARD UNIVERSITY AND

WESTERN RESERVE UNIVERSITY

\title{
A REMARK ON SEMI-SIMPLE LIE ALGEBRAS
}

GORDON BROWN

The following observation seems to be lacking in the literature.

THEOREM. The sum of the squares of the lengths of the roots of a semisimple Lie algebra is equal to the dimension of a Cartan subalgebra.

As usual the lengths of the roots are taken with respect to the nondegenerate Killing form $\operatorname{Tr}$ ad $x$ ad $y$.

Proof. No generality is lost in assuming the ground field algebraically closed.

Consider the matrix $M$ whose entries are the inner products $(\alpha, \beta)$, where $\alpha, \beta$ range over all the roots with respect to the Cartan subalgebra $H$. We have

$$
(\alpha, \beta)=\operatorname{Tr} \text { ad } h_{\alpha} \text { ad } h_{\beta}=\sum_{\gamma} \gamma\left(h_{\alpha}\right) \gamma\left(h_{\beta}\right)=\sum_{\gamma}(\alpha, \gamma)(\gamma, \beta)
$$

where $h_{\alpha}$ denotes the dual of $\alpha$; i.e., $\alpha(h)=\left(h_{\alpha}, h\right)$ for all $h$ in $H$. Thus $M^{2}=M$ and $\operatorname{Tr} M=\operatorname{rank} M$. Hence $\sum_{\alpha}(\alpha, \alpha)=\operatorname{dim} H$.

UNIVERSITY OF IlLINOIS

Received by the editors March 25, 1963. 